\title{
A W148R mutation in the human FOXD4 gene segregating with dilated cardiomyopathy, obsessive-compulsive disorder, and suicidality
}

\author{
PIERCARLO MINORETTI $^{1}$, MARIAROSA ARRA ${ }^{1}$, ENZO EMANUELE $^{1}$, VALENTINA OLIVIERI $^{1}$, \\ ALESSIA ALDEGHI ${ }^{1}$, PIERLUIGI POLITI ${ }^{2}$, VALENTINA MARTINELLI ${ }^{2}$, \\ SARA PESENTII ${ }^{2}$ and COLOMBA FALCONE ${ }^{1}$ \\ ${ }^{1}$ Interdepartmental Center for Research in Molecular Medicine (CIRMC); ${ }^{2}$ Department of Applied Health and \\ Behavioural Sciences, Section of Psychiatry, University of Pavia, Pavia, Italy
}

Received May 3, 2006; Accepted July 25, 2006

\begin{abstract}
The forkhead/winged helix box (FOX) gene family comprises at least 43 different genes encoding transcriptional factors with a highly conserved DNA-binding domain. To date, mutations in members of the FOX gene family have been causally linked to a variety of different human diseases. We describe a three-generation Albanian pedigree in which a complex phenotype consisting of dilated cardiomyopathy, obsessive-compulsive disorder, and suicidality is segregated with a missense mutation (W148R) in the human FOXD4 gene. This mutation disrupts an extremely highly conserved tryptophan residue in the forkhead domain of FOXD4, possibly resulting in reduced DNA binding capacity and altered transcriptional activity. Our present findings widen the spectrum of diseases associated with genetic aberrations in the forkhead gene family.
\end{abstract}

\section{Introduction}

The human forkhead/winged helix box (FOX) gene family comprises at least 43 different genes encoding transcription factors, which are crucially involved in the regulation of gene expression during ontogenesis (1). Members of the FOX family are characterized by the presence of a highly conserved DNA-binding forkhead domain, which is also termed 'winged-helix' due to its butterfly-like appearance (2). Mutations in different human FOX genes have been implicated in a variety of different genetic diseases (3-9),

Correspondence to: Professor Colomba Falcone, Interdepartmental Center for Research in Molecular Medicine (CIRMC), University of Pavia, Viale Taramelli 24, I-27100, Pavia, Italy

E-mail: ccirmc@unipv.it

Key words: candidate gene, forkhead, mutation analysis, cardiomyopathy, obsessive-compulsive disorder, suicidality including some neuropsychiatric phenotypes such as speech and language disorders (10) and schizophrenia (11).

The human FOXD4 is an intronless gene located at 9p24 $(1,12)$. It has been shown to be highly expressed in the heart and skeletal muscle, as well as in the adult and fetal brain $(13,14)$. In the present study, we describe a three-generation Albanian pedigree in which a complex phenotype consisting of dilated cardiomyopathy (DCM), obsessive-compulsive disorder (OCD), and suicidality is segregated with a missense mutation (W148R) in FOXD4.

We reasoned that FOXD4 could be a plausible biological candidate gene for the complex phenotype displayed by this family in light of its cerebral and cardiac expression $(13,14)$, and because it maps to a chromosomal region which has been previously linked to $\operatorname{OCD}(15,16)$.

\section{Patients and methods}

Case report. We describe an Albanian family (Family B, Fig. 1) showing a complex phenotype consisting of dilated cardiomyopathy (DCM), obsessive-compulsive disorder (OCD), and suicidality. The proband (III-1) was a 24 yearold male who was brought to our attention in May 2002 for dyspnea occurring during normal daily activities, as well as persistent chest discomfort upon exertion. He underwent a medical evaluation, including clinical history, physical examination, 12-lead electrocardiography, 2-dimensional echocardiography, and laboratory testing to screen for asymptomatic skeletal muscle disease. Echocardiographic examination revealed concentric left ventricular hypertrophy with diastolic dysfunction. Ejection fraction was estimated to be $33 \%$. Laboratory tests were negative for skeletal muscle disease. His personal history revealed eutocic delivery with a birth weight of 4,100 $\mathrm{g}$ and a normal psychomotor development. At the age of 16 , coinciding with personal crisis, moderate obsessive-compulsive symptomatology (touching objects and making others repeat phrases he claimed he had not understood) was shown, and a DSM-IV diagnosis of OCD was made by a private psychiatrist. Although he still complained to be haunted by some 


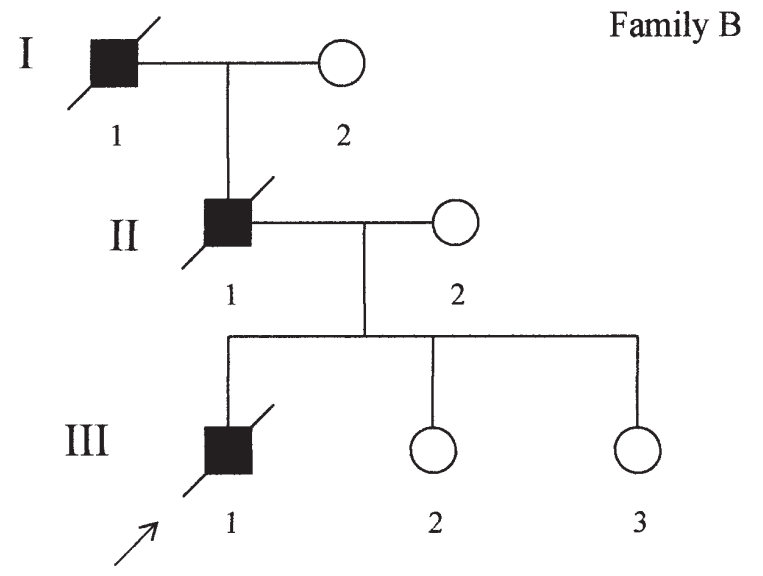

Figure 1. Pedigree of the affected family, including three generations. Unaffected individuals (open symbols), and suicidal subjects (filled symbols) are shown. Arrow indicates proband. The proband (III-1) and his father (II-1) harbored the FOXD4 mutation and had DCM and OCD. The proband's deceased paternal grandfather (I-1) was also reported to have committed suicide, but no information was available on his cardiac or psychiatric phenotype.

'unanswerable questions' and recognised them to be 'irrational', he reported not to receive any therapy for his obsessionality. He also rejected any possibility of referral to our psychiatry department for more in-depth evaluation. Similarly, though accepting to participate in molecular genetic analyses and scheduling for regular cardiac visits, he refused the possibility to be included in a heart transplant list. The patient's compliance to medical therapy for heart failure was poor, and he was soon lost to follow-up. In January 2003 , it was brought to our attention that he had committed suicide, leaving a suicide note referring to financial trouble.

The proband's father (II-1) displayed a very similar clinical phenotype. He manifested symptoms of dyspnea from the age of 45 years and was diagnosed with DCM. After experiencing severe economic difficulties, he began to worry excessively about money and expressed a number of financial concerns. He then started repeating obsessively what other people said and touching various objects. He committed suicide in February 2003 soon after his son's selfinflicted death.

Strikingly, the proband's deceased paternal grandfather (I-1) was also reported to have committed suicide after personal crisis. Unfortunately, no information or clinical chart was available on his potential cardiac or psychiatric symptoms, and there was no material for genetic analysis. The other members of this family, including the proband's mother (II-2) and the two younger sisters (III-2 and III-3), have had no history of cardiac or psychiatric disease.

Mutation analysis. Venous blood samples were drawn, after written informed consent, from the proband (III-1), younger sisters (III-2 and III-3), parents (II-1 and II-2), and paternal grandmother (I-2). Genomic DNA was purified by using a commercially available kit (Qiagen, Hilden, Germany) according to the manufacturer's protocol. The entire coding sequence of FOXD4 (GenBank Entry: NM_207305) was amplified by using two overlapping couples of primers designed with Primer3 (http://frodo.wi.mit.edu/cgibin/primer3/primer3_www.cgi). The primers used were as follows: FOXD4-1-sense, 5'-CTAGCGTTCCTGCTTCTTG C-3'; FOXD4-1-antisense, 5'-GTAGGTAGCGAGGAGG ATGC-3' (product size $910 \mathrm{bp}$; annealing temperature $62^{\circ} \mathrm{C}$ ); FOXD4-2-sense, 5'-CCCTTCCCTCTACCTGCTG-3'; FOXD4-2-antisense, 5'-AACCATTTTGCAGGGAACAG-3' (product size $804 \mathrm{bp}$; annealing temperature $59^{\circ} \mathrm{C}$ ). DNA template $(100 \mathrm{ng})$ were mixed in a solution containing $1 \mathrm{X}$ PCR buffer (100 mM Tris- $\mathrm{HCl} \mathrm{pH} 8.3 ; 500 \mathrm{mM} \mathrm{KCl}), 1.5$ $\mathrm{mM} \mathrm{MgCl} 2,50 \mu \mathrm{M}$ dNTPs, 10 pmol of each primer, and $2 \mathrm{U}$ of $P f u$ DNA polymerase (Promega, Madison, WI, USA) in a final reaction volume of $30 \mu \mathrm{l}$. The PCR reaction consisted of 35 cycles. PCR samples contained 5\% dimethyl sulfoxide to amplify both fragments. The products were purified by Wizard miniprep kit (Promega) and directly sequenced using an ABI Prism 377 DNA sequencer (Applied Biosystems, Foster City, CA, USA).

Genotyping of the W148R variant in unrelated DCM patients, OCD patients and healthy comparison subjects. To rule out the possibility that the W148R mutation in FOXD4 could be a polymorphic site, we genotyped three distinct cohorts consisting of 90 unrelated patients with DCM, 88 subjects with a DSM-IV diagnosis of OCD, as well as in 188 healthy control subjects. Genotyping was performed by means of PCR-RFLPs. PCR was carried out with the upstream primer 5'- TTTGGCACCGAGTTCAGG-3', and the downstream primer 5'-GTCCAGGCTCCAGTAGTTGC-3', in a final volume of $30 \mu \mathrm{l}$. PCR conditions consisted in an initial denaturation at $95^{\circ} \mathrm{C}$ for $90 \mathrm{sec}, 35$ cycles of a $94^{\circ} \mathrm{C}$ denaturing step for $30 \mathrm{sec}$, a $58^{\circ} \mathrm{C}$ annealing step for $45 \mathrm{sec}$, and a $72^{\circ} \mathrm{C}$ extension for $60 \mathrm{sec}$, followed by a final extension at $72^{\circ} \mathrm{C}$ for $5 \mathrm{~min}$, resulting in a $300 \mathrm{bp}$ fragment. PCR products $(10 \mu \mathrm{l})$ were digested using $2 \mathrm{U}$ of the endonuclease BstOI (Promega) for $16 \mathrm{~h}$ at $60^{\circ} \mathrm{C}$. The digested products were subjected to a $3 \%$ agarose gel electrophoresis and visualized under ultraviolet light after ethidium bromide staining. The wild-type $148 \mathrm{~W}$ allele yielded two distinctive fragments of 198 and 74 bp (as well as two short fragments of 23 and 5 bp, which were undetectable as discrete bands on agarose gels), while the mutant $148 \mathrm{R}$ allele yielded an unrestricted fragment of $272 \mathrm{bp}$ (as well as the two short fragments of 23 and $5 \mathrm{bp}$ ). All genotyping was done by laboratory personnel blinded to the case-control status of the samples, which included quality control samples for validation. Concordance for quality control samples was $100 \%$.

\section{Results}

Genomic sequencing of FOXD4 in the index patient (III-1) of the Albanian family revealed a heterozygous missense mutation in FOXD4 (W148R; 743T>C, Fig. 2A). The mutation was also found in the proband's father (II-1), but was not present in other unaffected members of this kindred (Fig. 2B). Thus the mutation segregated with the disease and was transmitted to the proband by the father.

The cross-species sequence alignment (Fig. 3) showed a strong conservation of the mutated tryptophan residue, which 

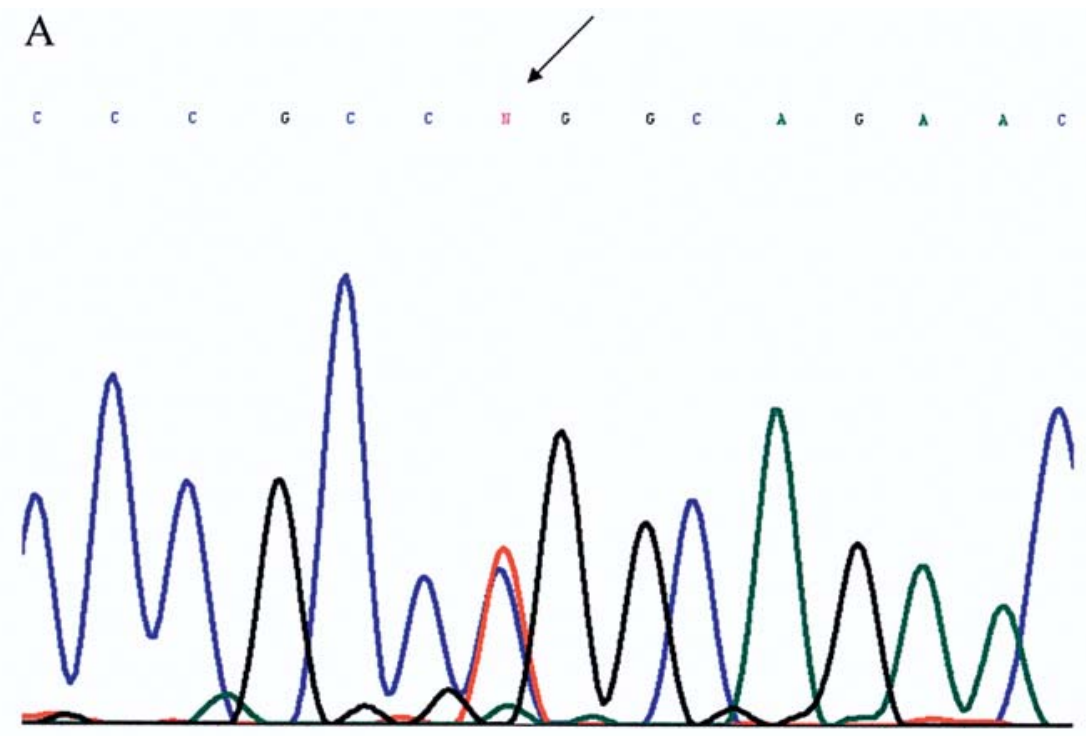

B

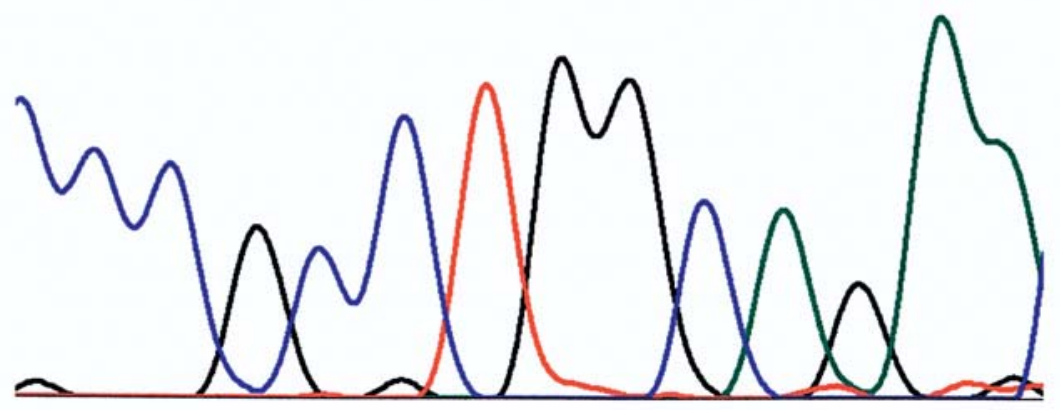

Figure 2. DNA sequence electrophoretogram (arrow, panel A) of the heterozygous mutation W148R (743T>C; GenBank entry: NM_207305) of FOXD4 in the proband (III-1). No equivalent mutation was detected in the proband's mother (II-2, panel B).

พ148

PAWONSI H. sapiens

PAWQNSI P. troglodytes

PAWONSI G. gorilla

PA用QNSI P. PYgmaeus

PAWQNSI M. musculus

PA用QNSI A. gambiae

PAWQNSI A. mellifera

Figure 3. Protein alignment of the mutation conserved across different species. is located in the highly-conserved DNA-binding fork-head region of FOXD4 (1). Importantly, this residue is also strikingly conserved in all members of the human FOX gene family (ref. 1; data not shown).

The $\mathrm{T}>\mathrm{C}$ mutation was not detected in 90 unrelated patients with DCM, 88 unrelated subjects with a DSM-IV diagnosis of OCD, or in the 376 normal control chromosomes.

\section{Discussion}

In this study, we identified a missense mutation (W148R) in the human FOXD4 associated with a complex phenotype consisting of dilated cardiomyopathy, obsessive-compulsive disorder, and suicidality in an Albanian family.

The present mutation is considered to be responsible for our patients' syndrome for the following reasons. First, the mutation is cosegregated with the disease in the present family with autosomal dominant transmission. Second, $743 \mathrm{~T}>\mathrm{C}$ was not found in 376 normal control chromosomes. Third, cross-species protein alignment showed a strong 
conservation of the mutated tryptophan residue in FOXD4. Notably, this amino acid is also strikingly conserved in the DNA-binding forkhead domain of all other human FOX genes (1), thereby indicating its potential structural or functional importance. These observations can be regarded as pieces of evidence indicating that the mutation detected in our family is pathogenic.

In future studies, it will be important to gain insights into the molecular mechanisms whereby this FOXD4 mutation alters protein function. Similar to other mutations in human FOX genes $(17,18)$, it is possible that the W148R variant could perturb the nuclear localization of FOXD4, or otherwise be severe enough to abolish FOXD4 DNA binding. This hypothesis can be tested by in vitro assays investigating nuclear localization of the mutant FOXD4, as well as its DNA binding capacity and transactivation potential.

The clinical features of our kindred are consistent with the tissue expression profile of FOXD4 in humans. Accordingly, we chose to study FOXD4 in our family because this gene is expressed at high levels both in the brain and the cardiac muscle $(13,14)$, as well as because FOXD4 maps to a region which has been previously linked to OCD $(15,16)$. On the other hand, of interest is also the observation that no muscular atrophy could be demonstrated in this family despite FOXD4 being abundantly expressed in skeletal muscle (13).

Our current findings raise a number of important points for both basic and clinical research. Firstly, the discovery of FOXD4 as a gene that may be involved in dilated cardiomyopathy and/or OCD will focus research efforts on searching for other mutations in this gene as possible causes for cardiac and/or psychiatric phenotypes. Although we were unable to find the W148R mutation in two distinct cohorts of unrelated patients with DCM or OCD, future studies investigating FOXD4 as a candidate gene in such disease conditions appear to be warranted. Confirming a role for FOXD4 in human disease may also provide opportunities for novel therapeutics and prevention, and contribute to a better understanding of how alterations in the FOX transcription factors may impact human diseases at a molecular level. The most important finding from this study possibly is the identification of FOXD4 as a potential candidate for suicidal behaviour. Although it is well-known that suicide runs in families and that genetic factors may contribute to suicidal behaviour (19), the great majority of molecular genetic studies has been focused on polymorphisms in serotonergic genes (20). Nonetheless, in line with previous observations (21), our present data indicate that molecular pathways other than the cerebral neurotransmission systems may play a pivotal role in the genetic vulnerability for suicidal behaviour.

In conclusion, we have shown that a mutation in FOXD4 affecting a highly conserved residue of the forkhead domain of this transcription factor cosegregates with dilated cardiomyopathy, obsessive-compulsive disorder, and suicidality in an Albanian kindred. This observation promises to offer novel insights into the molecular processes mediating cardiac hypertrophy, anxiety disorders and suicidality.

\section{Acknowledgements}

We gratefully acknowledge financial support from the AIPA ONLUS Foundation, Lecco, Italy.

\section{References}

1. Katoh M and Katoh M: Human FOX gene family (Review). Int J Oncol 25: 1495-1500, 2004.

2. Lantz KA and Kaestner KH: Winged-helix transcription factors and pancreatic development. Clin Sci 108: 195-204, 2005.

3. Mears AJ, Jordan T, Mirzayans F, et al: Mutations of the forkhead/winged-helix gene, FKHL7, in patients with AxenfeldRieger anomaly. Am J Hum Genet 63: 1316-1328, 1998.

4. Brice G, Mansour S, Bell R, et al: Analysis of the phenotypic abnormalities in lymphoedema-distichiasis syndrome in 74 patients with FOXC2 mutations or linkage to $16 \mathrm{q} 24$. J Med Genet 39: 478-483, 2002.

5. Castanet M, Park SM, Smith A, et al: A novel loss-of-function mutation in TTF-2 is associated with congenital hypothyroidism, thyroid agenesis and cleft palate. Hum Mol Genet 11: 2051-2059, 2002.

6. Semina EV, Brownell I, Mintz-Hittner HA, Murray JC and Jamrich M: Mutations in the human forkhead transcription factor FOXE3 associated with anterior segment ocular dysgenesis and cataracts. Hum Mol Genet 10: 231-236, 2001.

7. Crisponi L, Deiana M, Loi A, et al: The putative forkhead transcription factor FOXL2 is mutated in blepharophimosis/ ptosis/epicanthus inversus syndrome. Nat Genet 27: 159-166, 2001.

8. Harris SE, Chand AL, Winship IM, et al: Identification of novel mutations in FOXL2 associated with premature ovarian failure. Mol Hum Reprod 8: 729-733, 2002.

9. Frank J, Pignata C, Panteleyev AA, et al: Exposing the human nude phenotype. Nature 398: 473-474, 1999.

10. Vargha-Khadem F, Gadian DG, Copp A and Mishkin M: FOXP2 and the neuroanatomy of speech and language. Nat Rev Neurosci 6: 131-138, 2005.

11. Sanjuan J, Tolosa A, Gonzalez JC, et al: Association between FOXP2 polymorphisms and schizophrenia with auditory hallucinations. Psychiatr Genet 16: 67-72, 2006.

12. Humphray SJ, Oliver K, Hunt AR, et al: DNA sequence and analysis of human chromosome 9. Nature 429: 369-374, 2004.

13. Freyaldenhoven BS, Fried C and Wielckens K: FOXD4a and FOXD4b, two new winged helix transcription factors, are expressed in human leukemia cell lines. Gene 10: 131-140, 2002.

14. Wong A, Vallender EJ, Heretis K, et al: Diverse fates of paralogs following segmental duplication of telomeric genes. Genomics 84: 239-247, 2004.

15. Hanna GL, Veenstra-VanderWeele J, Cox NJ, et al: Genomewide linkage analysis of families with obsessive-compulsive disorder ascertained through pediatric probands. Am J Med Genet 114: 541-552, 2002.

16. Willour VL, Yao Shugart Y, Samuels J, et al: Replication study supports evidence for linkage to 9p24 in obsessive-compulsive disorder. Am J Hum Genet 75: 508-513, 2004.

17. Saleem RA, Banerjee-Basu S, Berry FB, Baxevanis AD and Walter MA: Analyses of the effects that disease-causing missense mutations have on the structure and function of the winged-helix protein FOXC1. Am J Hum Genet 68: 627-641, 2001.

18. Saleem RA, Banerjee-Basu S, Berry FB, Baxevanis AD and Walter MA: Structural and functional analyses of diseasecausing missense mutations in the forkhead domain of FOXC1. Hum Mol Genet 12: 2993-3005, 2003.

19. Marusic A: History and geography of suicide: could genetic risk factors account for the variation in suicide rates? Am J Med Genet C Semin Med Genet 133: 43-47, 2005.

20. Bondy B, Buettner A and Zill P: Genetics of suicide. Mol Psychiatry 11: 336-351, 2006.

21. Yanagi M, Shirakawa O, Kitamura N, et al: Association of 143-3 epsilon gene haplotype with completed suicide in Japanese. J Hum Genet 50: 210-216, 2005. 